\title{
THE WELL-POSEDNESS OF THE MIXED PROBLEM FOR ONE SYSTEM OF THERMOELASTICITY WITH A SINGULAR COEFFICIENT
}

\author{
AKBAR B. ALIYEV AND GUNAY R. GADIROVA
}

\begin{abstract}
Various problems of thermoelasticity are reduced to the mixed problem for a system of parabolic-hyperbolic equations. We consider the mixed problem for a system of thermoelastic plates with some nonLipschitz coefficients and investigate the well-posedness of the corresponding mixed problem in some functional spaces.
\end{abstract}

\section{Introduction}

In this paper we consider model equations for the thermoelastic plates

$$
\left\{\begin{array}{l}
u_{t t}+a(t) \Delta^{2} u+\delta \Delta \theta=f(t, x) \\
\theta_{t}-\Delta \theta-\delta \Delta u_{t}=g(t, x),
\end{array}\right.
$$

where $t \in[0, T], x \in \Omega, \delta \neq 0$, and $u=u(t, x), \theta=\theta(t, x)$ are deflection and the temperature difference, respectively. Here $\Omega$ is an open bounded domain in $R^{n}$ with smooth boundary $\Gamma=\partial \Omega$. Throughout the article, the letter $t$ in the indices of various functions denotes the derivative with respect to the variable $t$. Additionally, one has the boundary conditions

$$
u(t, x)=\Delta u(t, x)=\theta(t, x)=0, \quad t>0, x \in \Gamma
$$

and the initial conditions

$$
u(0, x)=u_{0}(x), \quad u_{t}(0, x)=u_{1}(x), \theta(0, x)=\theta_{0}(x), x \in \Omega .
$$

Various problems of thermoelasticity are reduced to the mixed problem for a system of parabolic-hyperbolic equations (see [14]). If the coefficients are sufficiently smooth, these problems have been studied by various authors in the works $[6,10$ $13,16-18,20,21]$. In the present paper we study the mixed problem (1.1)-(1.3) with non-smooth coefficient.

At first we recall some results concerning Cauchy problem for the hyperbolic equation

$$
\begin{aligned}
& u_{t t}-a(t) \Delta u=F(t, x), \quad t \in[0, T], x \in \Omega, \\
& u(0, x)=u_{0}(x), \quad u_{t}(0, x)=u_{1}(x), x \in \Omega,
\end{aligned}
$$

where $a(t) \geq a_{0}>0$

2010 Mathematics Subject Classification. 35B30, 35M40, 35L70, 35L99.

Key words and phrases. thermoelasticity, singular coefficient, mixed problem, well-posedness of the system of parabolic-hyperbolic equations. 
It is known that if $a(t)$ satisfies Lipschitz condition, then for any $u_{0} \in H^{s}(\Omega)$, $u_{1} \in H^{s-1}(\Omega)$ the problem(1.4)-(1.5) has a unique solution $u \in C\left([0, T] ; H^{s}(\Omega)\right)$ $\bigcap C^{1}\left([0, T] ; H^{s-1}(\Omega)\right)$ (see $\left.[15]\right)$. In the case when $a(t)$ does not satisfy the Lipschits condition, then corresponding assertion is no longer true (see [4]). In this case, the loss of smoothness happens, and therefore finding a class of wellposedness is a very important task. These problems were thoroughly studied for hyperbolic equations and interesting results were obtained (see, for example, [2-5, $9,19])$.

In the works $[6,10-13,16-18,20,21]$ and others, the Cauchy problem or the mixed problem for hyperboloic-parabolic systems were studied mainly when the coefficients in the principal part are smooth functions of the variable $t$. If the coefficients of the principal part of the hyperbolic-parabolic systems are not smooth, then the existence of the solution of the Cauchy problem or the mixed problem has not been investigated generally. In this direction we know only the papers [1] and [8]. In the work [8] the Cauchy problem for the system (1.1) is studied, in the case when $a(t)$ satisfies the logarithmic Lipschitz condition and for the appropriate Cauchy problem the energy estimate with loss was obtained.

We investigate the problem (1.1)-(1.3) with a singular coefficient $a(t)$. More exactly, we consider the case where the function $a(t)$ has no continuous derivative at the point $t=0$, and the function has some singularity as $t \rightarrow 0$. Depending on a singularity degree of $a(t)$, we define various correctness classes for the problem (1.1)-(1.3). Note that the Cauchy problem for thermoelastic systems with a similar singular coefficient was investigated in [1].

\section{Preliminary and main results}

Before formulating the obtained results we introduce some notations.

It is well known that, there exists an orthonormal basis $\left\{w_{k}\right\}$ in $L_{2}(\Omega)$, where $w_{k} \in \stackrel{0}{W}_{2}^{1}(\Omega)$ is an eigenfunction for the problem

$$
\begin{aligned}
& -\Delta w_{k}=\lambda_{k} w_{k} \text { in } \Omega, \\
& w_{k}=0 \text { on } \partial \Omega, k=1,2, \ldots .
\end{aligned}
$$

It is clear that $0<\lambda_{1} \leq \lambda_{2} \leq \lambda_{3} \leq \cdots$ and $\lim _{k \rightarrow \infty} \lambda_{k}=+\infty$ (see [7]).

For $\eta \geq 0$ we'll denote by $V(\eta)$ the functional space

$$
\begin{aligned}
& \qquad V(\eta)= \\
& =\left\{\varphi: \varphi \in L_{2}(\Omega),\|\varphi\|_{V(\eta)}=\left\{\sum_{k=1}^{\infty} \lambda_{k}^{\eta}\left|\varphi_{k}\right|^{2}\right\}^{1 / 2}<\infty, \varphi_{k}=\int_{\Omega} \varphi(x) w_{k}(x) d x\right\} \\
& \text { and } V=\bigcap_{\eta \geq 0} V(\eta) .
\end{aligned}
$$

For $s \geq 1$ we'll denote by $\gamma^{(s)}=\bigcap_{\beta>0} \gamma_{\beta}^{(s)}$ the Gevrey Space, where $f \in \gamma_{\beta}^{(s)}$ if $f \in L_{2}(\Omega)$ and

$$
\|f\|_{\gamma_{\beta}^{(s)}}=\left\{\sum_{k=1}^{\infty} \exp \left(\beta \lambda_{k}^{\frac{1}{s}}\right) f_{k}^{2}\right\}^{1 / 2}<+\infty
$$




$$
f_{k}=\int_{\Omega} f(x) w_{k}(x) d x
$$

First, let us assume that the following conditions are fulfilled:

(i) Let $a(t) \in C^{1}(0, T]$ and $a(t) \geq a_{0}, t \in(0, T]$, where $a_{0}>0$ is a positive constant.

(ii) Let $t \cdot\left|a^{\prime}(t)\right| \leq c, t \in(0, T]$, where $a^{\prime}(t)=\frac{d a(t)}{d t}$.

Under the conditions (i) and (ii) we show the well-posedness of the problem in the class of infinitely differentiable functions with respect to the space variables.

Theorem 2.1. Let a(t) satisfy (i) and (ii), then the problem (1.1)-(1.3) is well-posed in Von $[0, T]$. That is, for each initial data $u_{0}(x), u_{1}(x)$ and $\theta_{0}(x)$ from $V$ there exists a uniquely determined solution $(u, \theta) \in C^{1}([0, T], V) \times C([0, T], V)$.

Now we assume that the following condition is satisfied instead of (ii):

(ii*) Suppose that there exist $c_{1}, c_{2}>0$ such that, for all $t \in[0, T]$

$$
\begin{gathered}
t^{q}\left|a^{\prime}(t)\right| \leq c_{1}, \\
t^{p} a(t) \leq c_{2},
\end{gathered}
$$

where $q>1, p<q-1$ and $p \in[0,1)$.

In this case we prove that the problem (1.1)-(1.3) is well-posed in the class of Gevrey with respect to the space variables.

Theorem 2.2. Let $a(t)$ satisfy $(i)$ and $\left(i^{*}\right)$, then the problem (1.1)-(1.3) is well-posed in $\gamma^{(s)}$ on $[0, T]$ for all $s<\frac{q-p}{q-1}$, that is, for each initial data $u_{0}(x), u_{1}(x)$ and $\theta_{0}(x)$ from $\gamma^{(s)}$ there exists a uniquely determined solution $(u, \theta) \in C^{1}\left([0, T], \gamma^{(s)}\right) \times C\left([0, T], \gamma^{(s)}\right)$.

The proofs of Theorems 2.1 and 2.2 are carried out by the standard regularization method based on some energetic estimation.

Condition (ii) implies that $a(\cdot) \in L_{1}(0, T)$ and

$$
a(t) \leq c_{3}+c_{4} \ln \left(1+\frac{1}{t}\right)
$$

where $c_{3}, c_{4}>0$ (see $\left.[9]\right)$.

At first we give some auxiliary notations. We define for all $k=1,2, \ldots, t \in[0, T]$

$$
a_{r, k}(t)=\left\{\begin{array}{lll}
a(T) & \text { if } & \lambda_{k} \leq T^{-r}, \\
a\left(\lambda_{k}^{-1 / r}\right) & \text { if } & T^{-r}<\lambda_{k} \leq t^{-r}, \\
a(t) & \text { if } & \lambda_{k}>t^{-r}
\end{array}\right.
$$

where $r=s(q-1)$ if $q>1$ and $r=1$ if $q=1$. We define also

$$
A_{r, k}(t)= \begin{cases}\left|a_{r, k}(t)-a(t)\right| \cdot \lambda_{k} & \text { if } \quad \lambda_{k} \leq t^{-r} \\ \frac{\left|a^{\prime}(t)\right|}{a(t)} & \text { if } \quad \lambda_{k}>t^{-r}\end{cases}
$$

and

$$
h_{r, k}(\eta, t)=\left(1+\lambda_{k}^{2}\right)^{\eta} \exp \left(-\int_{0}^{t} A_{r, k}(s) d s\right) .
$$


Lemma 2.1. Let conditions (i) and (ii) be satisfied, then

$$
\begin{gathered}
a_{0} \leq a_{1, k}(t) \leq c_{5}+c_{6} \ln \lambda_{k}, k=1,2, \ldots, t \in[0, T], \\
\int_{0}^{t} A_{1, k}(\tau) d \tau \leq c_{7}+c_{8} \ln \lambda_{k},
\end{gathered}
$$

where $c_{i}>0, i=5, \ldots, 8$ are some constants independent of $k$.

Proof. Inequality (2.4) is obtained from inequality (2.1) and from definition of functions $a_{r, k}(t)$ (see (2.2)). Let us prove inequality (2.5).

Let $\lambda_{k}>T^{-1}$. By the definition of $A_{1, k}(t)$ we have

$$
\int_{0}^{t} A_{1, k}(s) d s \leq \int_{0}^{T}\left|a_{1, k}(s)-a(s)\right| \cdot \lambda_{k} d s \leq|a(T)|+\frac{1}{T}\|a(\cdot)\|_{L_{1}(0, T)} .
$$

Secondly, we consider the case $\lambda_{k} \leq T^{-1}$. From (2.3) and (2.4) we have

$$
\begin{gathered}
\int_{0}^{t} A_{1, k}(s) d s \leq \int_{0}^{T} A_{1, k}(s) d s \leq \int_{0}^{\lambda_{k}^{-1}}\left|a_{1, k}(s)-a(s)\right| \cdot \lambda_{k} d s+\int_{\lambda_{k}^{-1}}^{T} \frac{\left|a^{\prime}(t)\right|}{a(t)} d t \leq \\
\leq\left(c_{5}+c_{6} \ln \lambda_{k}\right)+\lambda_{k} \int_{0}^{\lambda_{k}^{-1}} a(t) d t+\frac{c_{1}}{a_{0}} \int_{\lambda_{k}^{-1}}^{T} \frac{d t}{t} \leq \\
\leq\left(c_{5}+c_{6} \ln \lambda_{k}\right)+\lambda_{k}^{-1} \int_{0}^{\lambda_{k}^{-1}}\left[c_{3}+c_{4} \ln \left(1+\frac{1}{t}\right)\right] d t+\frac{c_{1}}{a_{0}} \int_{\lambda_{k}^{-1}}^{T} \frac{d t}{t} \leq \\
\leq\left(c_{5}+c_{6} \ln \lambda_{k}\right)+c_{3}+c_{4} \lambda_{k}^{-1} \int_{0}^{\lambda_{k}^{-1}} \ln \left(1+\frac{1}{t}\right) d t+\frac{c}{a_{0}} \int_{\lambda_{k}^{-1}}^{T} \frac{d t}{t} \leq c_{7}+c_{8} \ln \lambda_{k} .
\end{gathered}
$$

The following lemma is proved in the same way.

Lemma 2.2. Let conditions (i) and $\left(i i^{*}\right)$ be satisfied, then

$$
\begin{gathered}
a_{0} \leq a_{r, k}(t) \leq c_{9} \lambda_{k}^{1+\frac{p}{r}} \\
\int_{0}^{t} A_{r, k}(\tau) d \tau \leq \eta \lambda_{k}^{\frac{q-1}{r}}+c_{\eta},
\end{gathered}
$$

where $\eta>0$ is an arbitrary positive number, $c_{\eta}>0$ and $c_{9}>0$ are some constants independent of $k$.

\section{Proof of Theorem 2.1}

We seek the solution of problem (1.1)-(1.3) in the form

$$
\begin{aligned}
& u=u(t, x)=\sum_{k=1}^{\infty} u_{k}(t) w_{k}(x), \\
& \theta=\theta(t, x)=\sum_{k=1}^{\infty} \theta_{k}(t) w_{k}(x),
\end{aligned}
$$

where $u_{k}(t)$ and $\theta_{k}(t)$ are the solutions of the Cauchy problem

$$
\left\{\begin{array}{l}
u_{k t t}(t)+a(t) \lambda_{k}^{2} u_{k}(t)-\delta \lambda_{k} \theta_{k}(t)=f_{k}(t), \\
\theta_{k t}(t)+\lambda_{k} \theta_{k}(t)+\delta \lambda_{k} u_{k t}(t)=g_{k}(t),
\end{array}\right.
$$


with the initial conditions

$$
u_{k}(0)=u_{0 k}, \quad u_{k t}(0)=u_{1 k}, \quad \theta_{k}(0)=\theta_{k 0},
$$

where

$$
\begin{aligned}
f_{k}(t) & =\int_{\Omega} f(t, x) w_{k}(x) d x, \quad g_{k}(t)=\int_{\Omega} g(t, x) w_{k}(x) d x, \\
u_{0 k} & =\int_{\Omega} u_{0}(x) w_{k}(x) d x, \quad u_{1 k}=\int_{\Omega} u_{1}(x) w_{k}(x) d x .
\end{aligned}
$$

Let us define the weighted energetic function in the following way

$$
E(\eta, t)=\sum_{k=1}^{\infty} E_{k}(\eta, t),
$$

where

$$
E_{k}(\eta, t)=\left[u_{k t}^{2}(t)+\left(1+a_{r, k}(t) \lambda_{k}^{2}\right) \cdot u_{k}^{2}(t)+\theta_{k}^{2}(t)\right] \cdot h_{r, k}(\eta, t) .
$$

In view of (3.3) after simple transformations we get

$$
\begin{gathered}
\frac{d E_{k}(\eta, t)}{d t}=\left[2 u_{k t t}(t) \cdot u_{k t}(t)+2 \theta_{k}(t) \cdot \theta_{k t}(t)+\right. \\
\left.+a_{r, k}(t) \lambda_{k}^{2} \cdot u_{k}^{2}(t)+\left(1+a_{r, k}(t) \lambda_{k}^{2}\right) \cdot 2 u_{k t}(t) \cdot u_{k}(t)\right] \cdot h_{k}(\eta, t)- \\
-E_{k}(\eta, t) \cdot A_{r, k}(t) .
\end{gathered}
$$

By (3.1) it follows from relation (3.4) that

$$
\begin{aligned}
& \frac{d E_{k}(\eta, t)}{d t}=\left[\left[1+\left(a_{r, k}(t)-a(t)\right) \lambda_{k}^{2}\right] \cdot h_{r, k}(\eta, t) u_{k}(t) u_{k t}(t)+\right. \\
& \left.+a_{r, k t}(t) \cdot \lambda_{k}^{2} u_{k}^{2}(t)-\lambda_{k} \cdot \theta_{k}^{2}(t)\right] \cdot h_{r, k}(\eta, t)-E_{k}(\eta, t) A_{r, k}(t)+ \\
& +2\left[u_{k t}(t) f_{k}(t)+\theta_{k}(t) g_{k}(t)\right] \cdot h_{r, k}(\eta, t) .
\end{aligned}
$$

Since

$$
2 u_{k t}(t) f_{k}(t) \leq u_{k t}^{2}(t)+f_{k}^{2}(t) \text { and } 2 \theta_{k}(t) g_{k}(t) \leq \theta_{k}^{2}(t)+g_{k}^{2}(t),
$$

from (3.5) we obtain the following inequality

$$
\begin{aligned}
& \frac{d E_{k}(\eta, t)}{d t} \leq {\left[\left[1+\left(a_{r, k}(t)-a(t)\right) \lambda_{k}^{2}\right] \cdot h_{r, k}(\eta, t) \cdot 2 u_{k}(t) u_{k t}(t)+\right.} \\
&\left.+a_{r, k t}(t) \cdot \lambda_{k}^{2} u_{k}^{2}(t)-\lambda_{k} \cdot \theta_{k}^{2}(t)\right] \cdot h_{r, k}(\eta, t)-E_{k}(\eta, t) A_{r, k}(t)+ \\
&+\left[u_{k}^{2}(t)+\theta_{k}^{2}(t)+f_{k}^{2}(t)+g_{k}^{2}(t)\right] \cdot h_{r, k}(\eta, t) .
\end{aligned}
$$

If $\lambda_{k}>t^{-r}$, then by virtue of (2.2) and (2.3) we get

$$
a_{r, k}(t)-a(t)=0, \quad A_{r, k}(t)=\frac{\left|a^{\prime}(t)\right|}{a(t)},
$$

and hence

$$
\begin{aligned}
& a_{r, k t}(t) \lambda_{k}^{2} u_{k}^{2}(t) h_{r, k}(\eta, t)-E_{k}(\eta, t) A_{r, k}(t) \leq \\
& \leq\left(a_{r, k t}(t)-\left|a_{r, k t}(t)\right|\right) \lambda_{k}^{2} u_{k}^{2}(t) h_{r, k}(\eta, t) \leq 0 .
\end{aligned}
$$

By virtue of (3.7) and (3.8), it follows from (3.6) that

$$
\frac{d E_{k}(\eta, t)}{d t} \leq C\left[E_{k}(\eta, t)+\left(f_{k}^{2}(t)+g_{k}^{2}(t)\right) \cdot h_{r, k}(\eta, t)\right],
$$

where $C>0$ is independent of $k$. 
If $\lambda_{k} \leq t^{-r}$, then by (2.2) and (2.3) we have

$$
a_{r, k t}(t)=0, A_{r, k}(t)=\left|a_{r, k}(t)-a(t)\right| \cdot \lambda_{k},
$$

and consequently,

$$
\left(a_{r k}(t)-a(t)\right) \lambda_{k}^{2} u_{k}(t) . u_{k t}(t) h_{r, k}(\eta, t)-A_{r, k}(t) E_{k}(\eta, t) \leq 0 .
$$

Therefore, we again obtain (3.9), from which it follows that

$$
\frac{d E(\eta, t)}{d t} \leq C[E(\eta, t)+\Phi(\eta, t)]
$$

where $\Phi(\eta, t)=\sum_{k=1}^{\infty}\left[f_{k}^{2}(t)+g_{k}^{2}(t)\right] \cdot h_{k}(\eta, t)$.

Using (3.2) and applying the Gronwall's inequality from (3.10), we get

$$
E(\eta, t) \leq M_{T}\left[E(\eta, 0)+\int_{0}^{T} \Phi(\eta, \tau) d \tau\right], t \in[0, T],
$$

where $M_{T}>0$ is a constant independent of $t$.

In the case $r=1$ from Lemma 2.1 it follows that for any $\eta \geq 0$ and $\varepsilon>0$

$$
E(\eta, t) \leq \mathrm{H}(\eta+\varepsilon, t),
$$

where

$$
\mathrm{H}(\alpha, t)=\sum_{k=1}^{\infty}\left(1+\lambda_{k}^{2}\right)^{\alpha}\left[u_{k t}^{2}(t)+\lambda_{k}^{2} u_{k}^{2}(t)+\theta_{k}^{2}(t)\right] .
$$

By (3.12) and (3.13) there exists $M_{1}>0$ such that

$$
e^{-\int_{0}^{t} A_{1, k}(s) d s} \geq M_{1} \lambda_{k}^{-c_{8}} .
$$

Hence in the case $r=1$ there exist $N>0$ and $M_{2}>0$ such that for all $t \in[0,1]$

$$
E(\eta, t) \geq M_{2} \cdot \mathrm{H}(\eta-N, t) .
$$

From (3.12) and (3.14) it follows that the Cauchy problem (1.1)-(1.3) is wellposed in $C^{1}([0, T] ; V) \times C([0, T] ; V)$.

\section{Proof of Theorem 2.2}

Let $1<s<\frac{q-p}{q-1}$.

At first we introduce some notations:

$$
\Lambda_{k}(\beta, s, t)=\mathrm{E}_{k}(0, t) \exp \left(\beta \lambda_{k}^{1 / s}\right), \Lambda(\beta, s, t)=\sum_{k=1}^{\infty} \Lambda_{k}(\beta, s, t),
$$

where $\beta>0,1<s<\frac{q-p}{q-1}$.

In the same way as we obtained inequality (3.11) we can show that, there exists a constant $M_{T, 1}>0$ which independent of $t$ such that

$$
\Lambda(\beta, s, t) \leq M_{T, 1}\left[\Lambda(\beta, s, 0)+\int_{0}^{T} \mathrm{P}(\beta, s, \tau) d \tau\right], t \in[0, T],
$$

where $\mathrm{P}(\beta, s, t)=\sum_{k=1}^{\infty}\left[\left|f_{k}(t)\right|^{2}+\left|g_{k}(t)\right|^{2}\right] \cdot h_{k}(0, t) \exp \left(\beta \lambda_{k}^{1 / s}\right)$. 
For $r>1$, it follows from Lemma 2.2 that there exist $\mathrm{M}_{1}, \mathrm{M}_{2}>0$ such that for every $\beta>0$ and $\varepsilon>0$ the following inequalities hold:

$$
\begin{gathered}
\mathrm{M}_{1}\left[\left\|u_{t}(t, \cdot)\right\|_{\gamma_{\beta-\varepsilon}^{(s)}}+\|u(t, \cdot)\|_{\gamma_{\beta-\varepsilon}^{(s)}}+\|\theta(t, \cdot)\|_{\gamma_{\beta-\varepsilon}^{(s)}}\right] \leq \Lambda(\beta, s, t) \leq \\
\leq \mathrm{M}_{2}\left[\left\|u_{t}(t, \cdot)\right\|_{\gamma_{\beta+\varepsilon}^{(s)}}+\|u(t, \cdot)\|_{\gamma_{\beta+\varepsilon}^{(s)}}+\|\theta(t, \cdot)\|_{\gamma_{\beta+\varepsilon}^{(s)}}\right], t \in[0, T], \\
\mathrm{M}_{1}\left[\|f(t, \cdot)\|_{\gamma_{\beta-\varepsilon}^{(s)}}+\|g(t, \cdot)\|_{\gamma_{\beta-\varepsilon}^{(s)}}\right] \leq \\
\leq \mathrm{P}(\beta, s, t) \leq \mathrm{M}_{2}\left[\|f(t, \cdot)\|_{\gamma_{\beta+\varepsilon}^{(s)}}+\|g(t, \cdot)\|_{\gamma_{\beta+\varepsilon}^{(s)}}\right], t \in[0, T] .
\end{gathered}
$$

Now, using (4.2) and (4.3), we can show that there exists $\mathrm{M}_{3}>0$ such that for any $\beta>0$ and $\varepsilon>0$

$$
\begin{gathered}
\left\|u_{t}(t, \cdot)\right\|_{\gamma_{\beta-\varepsilon}^{(s)}}+\|u(t, \cdot)\|_{\gamma_{\beta-\varepsilon}^{(s)}}+\|\theta(t, \cdot)\|_{\gamma_{\beta-\varepsilon}^{(s)}} \leq \\
\leq \mathrm{M}_{3}\left[\left\|u_{0}(\cdot)\right\|_{\gamma_{\beta+\varepsilon}^{(s)}}+\left\|u_{1}(\cdot)\right\|_{\gamma_{\beta+\varepsilon}^{(s)}}+\left\|\theta_{0}(\cdot)\right\|_{\gamma_{\beta+\varepsilon}^{(s)}}\right]+ \\
+\mathrm{M}_{3} \int_{0}^{T}\left[\|f(\tau, \cdot)\|_{\gamma_{\beta+\varepsilon}^{(s)}}+\|g(\tau, \cdot)\|_{\gamma_{\beta+\varepsilon}^{(s)}}\right] d \tau, \quad t \in[0, T] .
\end{gathered}
$$

From (3.12) and (3.14) it follows that the Cauchy problem (1.1)-(1.3) is wellposed in $C^{1}\left([0, T] ; \gamma^{(s)}\right) \times C\left([0, T] ; \gamma^{(s)}\right)$.

\section{References}

[1] A.B. Aliev and G. R. Gadirova, The well-posedness of the Cauchy problem for a system of thermoelasticity with singular coeficients, Proc. Inst. Math. Mech. Natl. Acad. Sci. Azerb., 43 (2017), No. 2, 233-239.

[2] A.B. Aliev and G.D. Shukurova, Well-posednes of the Cauchy problem for hyperbolic equations with non-lipschitz coeficients, Abstr. Appl. Anal. 2009, Article ID 182371, 15 pages, doi:10.1155/2009/182371,15.

[3] M. Cicognani, F. Colombini, Modulus of continuity of the coefficients and loss of derivatives in the strictly hyperbolic Cauchy problem, J. Differential Equations 221 (2006), No.1, 143-157.

[4] F. Colombini, E. De Giorge, S. Spagnolo, Existence et uniqute des solutions des equations hyperboluques du second orde a coefficients no dependant, Comptes Rendus de l'Academie des Sciences, 286 (1978), 1045-1048.

[5] F. Colombini, D. Del Santo, T. Kinoshita, Well-posedness of the cauchy problem for hyperbolic equations with non Lipschitz coefficients, Annali della Scuola Normale Superiore di Pisa 1 (2002), No. 2, 327-358.

[6] C.M. Dafermos, On the existence and the asymptotic stability of solutions to the equations of linear thermoelasticity, in: Arch. Rational Mech. Anal. 29 (1968), 241271.

[7] L.C. Evans, Partial differential equations, Graduate Studies in Mathematics, 19, AMS, (1998), $662 \mathrm{pp}$.

[8] G.R. Gadirova, Loss of smoothness of solution of a hyperbolic-parabolic system with singular coefficient, Trans. NAS Azerb., XXXIV (2014), No. 4, 37-44.

[9] F. Hirosawa, Loss of regularity for second order hyperbolic equations with singular coefficients, Osaka J. Math. 42 (2005), No. 4, 767-790. 
[10] W.J. Hrusa, M.A. Tarabek, On smooth solutions of the Cauchy problem in onedimensional nonlinear thermoelasticity, Quart. Appl. Math. 47 (1989), 631-644.

[11] L. Hsiao, S. Jiang, Nonlinear hyperbolic-parabolic coupled systems, in Handbook of Differential Equations, Evolutionary Equations (Dafermos, C.M. \& Feireisl, E. (Eds.)), Vol. 1, Elsevier (2004), 287-384.

[12] S. Jiang, Global smooth solutions to a one-dimensional nonlinear thermoviscoelastic model, Adv. Math Sci. Appl. 7 (1997), No.2 771-787.

[13] S. Jiang, J.E. Mu noz Rivera and R. Racke, Asymptotic stability and global existence in thermoelasticity with symmetry, Quart. Appl. Math. 56 (1998), 259-275.

[14] V. D. Kupradze, T. G. Gegelia, M. O. Basheleishvili, and T. V. Burchuladze, Threedimensional problems of the mathematical theory of elasticity and thermoelasticity, Applied Mathematics and Mechanics, vol. 25, North-Holland, (1979), 929 pp.

[15] J.L. Lions and E. Madgenes, Non Homogeneous Boundary value Problems and Applications, Springer Verlaq, (1973), 372 pp.

[16] G. Ponce and R. Racke, Global existence of small solutions to the initial value problem for nonlinear thermoelasticity, J. Differential Equations 87 (1990), No. 1, 70-83.

[17] R. Racke, Y. Shibata, S. Zheng, Global solvability and exponential stability in onedimensional nonlinear thermoelasticity, Quart. Appl. Math. 51 (1993), No. 4, 751-763.

[18] R. Racke, Y.G. Wang, Nonlinear well-posedness and rates of decay in thermoelasticity with second sound, J. Hyperbolic Differ. Equ. 5 (2008), No. 1, 25-43.

[19] M. Reissig, About strictly hyperbolic operators with non-regular coefficients, Pliska Stud. Math. Bulgar. 15 (2003), 105-130.

[20] Y.G. Wang, L. Yang, $L p \rightarrow L q$ decay estimates for Cauchy problems of linear thermoelastic systems with second sound in three dimensions, Proc. R. Soc. Edinburgh, Sec. A 136 (2006), No. 1, 189-207.

[21] L. Yang, Y G. Wang, Well-posedness and decay estimates for Cauchy problems of linear thermoelastic systems of type III in 3-D, Indiana Uinv. Math. J 55 (2006), No. 4, 1333-1361.

Akbar B. Aliyev

Institute of Mathematics and Mechanics National Academy of Sciences of Azerbaijan, Baku, AZ 1141, Azerbaijan

Azerbaijan State Oil and Industry University, Baku, Az 1010, Azerbaijan

E-mail address: alievakbar@gmail.com

Gunay R. Gadirova

Institute of Mathematics and Mechanics National Academy of Sciences of Azerbaijan, Baku, AZ 1141, Azerbaijan

Guba branch of Azerbaijan State Pedagogical University

E-mail address: gunayqedirova@inbox.ru

Received: March 3, 2021; Revised: July 16, 2021; Accepted: July 28, 2021 